Jacques Guilhaumou

CNRS/ENS-LSH Lyon

\title{
La langue politique et la Révolution française ${ }^{1}$.
}

\section{Introduction}

Au cours du débat à l'Assemblée Nationale sur le veto royal, les premiers jours de septembre 1789, Rabaud Saint-Etienne s'inquiète de «la pauvreté de notre langue pour exprimer les idées politiques absolument neuves pour la masse de la nation $»^{2}$. Sieyès, présent à ce débat, approuve l'intervention de son collègue, tout en considérant qu'il a été, dès 1788 et tout particulièrement avec Qu'est-ce que le Tiers-Etat? le principal inventeur de "la nouvelle langue politique », selon sa propre expression (Guilhaumou, 2002a).

Au terme de son parcours intellectuel, il revient sur cette question au cours des années 1810 dans les termes suivants :

" Ceux qui forment une science dans laquelle doivent se ranger et se fondre des foules d'idées qui existent déjà bien ou mal dans la langue usuelle sont bien embarrassés. Ces idées ou plutôt les mots qui les expriment sont sujets à de nombreuses acceptions, pour ainsi dire mobiles. Les nuances nécessaires manquent de signes précis et propres. Les notions elles-mêmes ont été mal faites dans l'origine, ou ont été altérées par le temps. Et cependant il faut parler, observer les faits exacts, les lier entre eux, les analyser, en tirer des notions générales, retrouver ces notions dans des conséquences rigoureuses, enfin raisonner. Tout cela suppose qu'on crée une langue nouvelle avec des matériaux confusément épars, et qui résistent à recevoir un emploi déterminé $»^{3}$.

Un tel processus de création d'une «langue nouvelle» de «la science politique " est le propre de la Révolution française. Nous considérons

\footnotetext{
${ }^{1}$ Cet article dresse un bilan des travaux récents sur la culture politique de la Révolution française d'un point de vue discursif. Il actualise une recherche que nous menons depuis une trentaine d'années, mais en la situant plus nettement dans la perspective de l'histoire langagière des concepts (2006b). Nous reprenons ainsi, en les associant et en les précisant, les synthèses discursives que nous avons présentées aux colloques sur La Révolution française au carrefour des recherches (MMSH, Université de Provence) et sur La Révolution à l'œuvre (IHRF, Paris I) publiés respectivement par Martine Lapied et Christine Peyrard aux Publications de l'Université de Provence, et Jean-Clément Martin aux Presses Universitaires de Rennes, ainsi que dans le volume collectif dirigé par Raymonde Monnier (2004).

${ }^{2}$ Archives Parlementaires, tome VIII, p. 68 [texte disponible sur Gallica].

${ }^{3}$ Note manuscrite intitulée Onéologie, Archives Nationales, 284 AP 5 (3).
} 
présentement qu'il importe d'en confronter la connaissance que nous en avons (Guilhaumou, 1989a) aux avancées historiographiques récentes, avec une attention particulière à la manière dont s'y déploie, entre usages réflexifs des acteurs et concepts des historiens, les notions-concepts.

Constatons d'abord que l'événement révolutionnaire, contexte principal de la créativité politique, n'est pas exempt d'un arrière plan social. C'est à ce titre que nous sommes d'emblée confronté à un univers de notions qui correspondent à une manière usuelle d'interpréter, chez les êtres humains, leur expérience du monde de façon quasi-naturelle. Il s'agit du terrain des "mœurs", selon l'expression usitée à l'époque, mais que Sieyès qualifie de façon éphémère, mais significative par le terme de « sociologie » (Guilhaumou, 2006a).

Ce terrain commun de la langue usuelle, descriptible sous la notion-concept d'utilité, est d'un constant secours: il constitue en effet «le socle sociologique » du nécessaire processus de généralisation de la langue politique, dans la mesure où il permet aux notions d'acquérir une dimension conceptuelle sur la base d'une systématisation abstraite, tout en conservant un lien à l'observation empirique des faits. Une telle présence permanente de «l'organisme social » confère au domaine de «l'art social », où se fabrique les notions artificielles de la langue politique, une tournure réaliste par la présence singulière des expériences individuelles.

Certes les révolutionnaires héritent des Lumières le principe de la connexion entre la réalité et le discours comme seul moyen d'exercer un contrôle sémiotique, donc de fixer des règles rationnelles d'usage des mots, dans le domaine de l'expérience humaine (Formigari, 1993). Mais ils évoluent dans un univers de notions qui sont à la fois usages et concepts, constituant ainsi, par la multiplication des mises en argument, un maquis de notions marquées contextuellement. Qui plus est, le chercheur actuel se démarque à son tour de cette profusion par souci de clarté, tout en multipliant, contre son gré, les " usages dispersants et mutants » selon l'expression de Maurice Tournier ${ }^{4}$ de ce nous appelons les notions-concepts.

Evoluer dans le monde des "usages conceptuels ", devenant " concepts en usage " n'est donc pas une affaire aisée. Ferdinand Brunot (1967), avec son Histoire de la langue française, avait ouvert la voie. Les travaux de lexicologie politique de ces trente dernières années l'ont singulièrement élargi tant sur le plan des matériaux que des problématiques. Retraçons rapidement les principales étapes éditoriales de ce cheminement à l'intérieur des études sur les notions-concepts de la Révolution française.

Alors que se met en place en Allemagne la vaste entreprise, toujours en cours de publication, du Handbuch politisch-sozialer Grundbegriffe in Frankreich (1680-1820) sous la direction de Hans-Jürgen Lüsebrink, Rolf Reichardt et

\footnotetext{
${ }^{4}$ Dans sa Préface à Des notions-concepts en révolution, sous la dir. de J. Guilhaumou et R. Monnier (2003).
} 
Eberhard Schmitt, c'est d'abord en France le temps du projet par la publication en 1987 par l'équipe « $18^{\text {ème }}$ et Révolution » de l'ENS de Saint-Cloud du second fascicule du Dictionnaire des usages socio-politiques du français entre 1770 et 1815 sur les notions-concepts, où se formule, sous notre plume, l'objectif de rendre compte de mots à valeur notionnelle souvent cités, mais plutôt méconnus dans leur réalité discursive. Cette perspective est développée par Hélène Dupuy dans sa brève intervention au colloque bilan de l'Institut d'Histoire de la Révolution française (Vovelle, 1991) sous le titre significatif, "Pour une redécouverte du politique à travers les notions-concepts ». La publication en 1995 du colloque de Saint-Cloud sur les Langages de la révolution (1770-1815) accentue l'importance des études sur l'expression langagière des concepts, toujours sous l'égide de "l'équipe $18^{\text {ème }}$ et Révolution" du laboratoire de lexicologie politique de l'ENS de Saint-Cloud, véritable cheville ouvrière de l'ensemble de cette entreprise française. Enfin le tout récent volume (2003), sur Des notions-concepts en révolution, sous la direction de Raymonde Monnier et moi-même marque une nouvelle étape de la recherche par l'établissement d'une liaison régulière, dans le cadre du réseau international « History of Political and Social Concepts Group ", entre l'histoire linguistique des usages conceptuels pratiquée désormais par les chercheurs français, l'histoire $d u$ discours, initié dans les années 1970 par John Pocock et Quentin Skinner au sein de la recherche anglophone, et l'histoire sémantique, autour de Reinhart Koselleck et Rolf Reichardt, dans le monde allemand ${ }^{5}$. Enfin, le bilan proposé relève de la problématique de l'événement linguistique, c'est-à-dire nous renvoie à la part de réflexivité langagière au sein des événements révolutionnaires, dans la perspective développée de notre première publication (1989) sur La langue politique et la Révolution française à notre ouvrage le plus récent (2006b) sur Discours et événement.

Cependant nous nous limitons, à l'intérieur de ce vaste champ discursif, à l'univers des notions-concepts spécifiques de la langue politique de la Révolution française, tout en le contextualisant selon les moments de la Révolution française, tels que nous les avons mis en évidence, de concert avec Françoise Brunel (1991). A la présentation des résultats de l'analyse discursive des notions en usage s'ajoute donc un souci de rendre compte des configurations successives de la synthèse politique sur le terrain proprement discursif. Parcours déséquilibré par le fait de la répartition très inégale des travaux lexicologiques selon chaque moment, et qui nous incite à marquer un intérêt particulier pour le moment inaugural des années 1770-1780, peu connu et qualifié à tort de prérévolutionnaire.

\footnotetext{
5 Voir l'ouvrage collectif sous la direction de Hans Erich Bödeker (2002), et plus particulièrement notre intervention sur «L'histoire linguistique des usages conceptuels à l'épreuve des événements linguistiques » p. 123-158.
} 


\section{I - Le moment proto-politique : les années 1770-1780.}

Le premier moment historique que nous proposons de caractériser constitue l'objet principal de nos recherches actuelles, dans la mesure où il a été longtemps négligé tant par les historiens que par les lexicologues. Il s'agit des années 1770-1780 que nous qualifions de moment proto-politique - ou néopolitique - en référence à l'apparition, dans cette période, d'un pouvoir ontologique de thématiser la réalité sociale à l'aide d'objets notionnels inédits, sur la base d'un "socle sociologique " apte à permettre l'émergence d'une nouvelle langue politique.

A vrai dire, ce "socle sociologique » s'est constitué, avant de prendre le nom sociologie sous la plume de Sieyès, dans la transition, perceptible dès les années 1770 , d'une vision essentialiste de la société, qui laisse impensé le peuple en acte, à des considérations sur l'ordre social en termes d'expérience, donc où des formes d'accord, voire de désaccord, s'instaure entre les élites et le peuple au plus grand profit de la mise en évidence de dispositifs populaires d'agir. Ainsi, interrogeant le devenir du nom de peuple dans les écrits littéraires et les témoignages judiciaires, Deborah Cohen (2004) précise ce qu'il en est d'un tel pragmatisme des constructions identitaires populaires et de leur articulation avec le discours savant. Ainsi s'ouvre un espace des possibles à l'expérience du tout social, immédiatement relayé par un espace du pensable dans ce moment nominaliste où «le pouvoir ontologique de la société devient une thématique politique " selon l'heureuse expression de la sociologue Laurence Kaufmann (2000). C'est cet espace foncièrement nominaliste que nous souhaitons caractériser ici à grands traits.

Ce moment nominaliste correspond en premier lieu au temps d'imposition de l'opinion publique, non seulement sous la forme d'une réalité empirique par la multiplication des espaces de sociabilité (salons, académies, cafés, etc.) où s'exerce un usage public $\mathrm{du}$ raisonnement, mais aussi de manière " artificialiste », c'est-à-dire avec une teneur à la fois plus juridique, plus individualiste, plus politique, en annonce du modèle national et législatif de 1789 (Kaufmann, 2004).

Ainsi, une autre notion-concept est révélatrice du "poids sociologique » de ce moment, celui d'individu. Pris dans l'utile, l'individu est définit par sa capacité propre d'autoconservation, donc au plus près de nature. Mais ses usages marquent aussi un déplacement de préoccupations populationnistes, chez les économistes, vers des considérations sur la liberté individuelle, par un phénomène d'expansion de sa nature à l'ensemble de la société, mais avec ses limites propres du côté du politique (Viguier, 1989).

C'est pourquoi s'opère, au-delà de ce positionnement sociologique, un travail de l'esprit politique, sous l'égide des figures complémentaires de l'observateur philosophe et du législateur, qui relève d'emblée d'un nominalisme politique 
et prend le nom d' "art social» (Kaufmann, Guilhaumou, 2003). Ici, le législateur, qui conçoit et réalise l'ordre social, le fait au titre d'un " art social » qui prend en compte la seule réalité de l'individu empirique.

Nous considérons que ce travail de l'esprit politique trouve un contexte favorable au milieu des années 1770 et peut ainsi faire rupture au cours des années 1780. Ainsi se met en place un dispositif novateur de descriptions et d'actions sociales, qualifié par Sieyès de "métaphysique politique " (Guilhaumou, 2002).

Ce nouveau dispositif a d'abord été situé discursivement par Keith Baker (1990) à l'intérieur d'une tripartition entre le discours judiciaire des parlementaires, le discours administratif des réformateurs et le discours de la volonté des philosophes politiques, de Rousseau à Sieyès. Ainsi se précise la prééminence progressive de la thématique politique. Cependant les travaux de Daniel Gordon (1994), complexifie une telle transition vers la domination du politique par la prise en compte des conséquences de l'auto-institution du social. En effet, alors que la notion de société civile a acquis une place singulière dans le nouveau discours social (Branca-Rosff, Guilhaumou, 2003), la " socialité » peut déjà se décliner selon divers registres égalitaires, au titre de l'usage novateur et généralisé de l'adjectivation « social(e) » légitimée par l'édition de l'Encyclopédie de 1765, sans qu'aucune prédétermination à la voie de l'action politique n'existe. Comme l'a montré par ailleurs un collectif d'historiens autour de Dale Van Kley (1994), il est possible de repérer la présence et le développement dans les corps et les communautés d'Ancien Régime des composantes essentielles de la notion de liberté : l'égalité, la liberté-protection et la liberté-participation, certes au sein d'une hiérarchie sociale toujours aussi inégalitaire, mais qui peut permettre la formation d'un consensus civique, une fois levée l'hypothèque de la monarchie absolue.

A vrai dire, le trajet historique qu'il convient ici de prendre compte est celui qui nous mène de l'échec des réformateurs de la monarchie, en 1775-1776, face à la résistance d'un peuple raisonnable selon l'expression de Mably au climat pamphlétaire des années 1788-1789 où s'élabore, selon les historiens, une synthèse nationale qui élimine la référence passéiste aux faits historiques.

De fait, les années 1775-1776 sont d'abord marquées par l'essai avorté de Turgot d'établir la liberté du commerce des grains qui se heurte au mouvement de «la guerre des farines» (Bouthon, 1993). L'illusion de rendre le gouvernement «maître des subsistances" se dissipe rapidement face à la traduction de l'émeute par les observateurs éclairés en un récit annonciateur d'événements politiques. Le " peuple raisonnable » est ici l'ensemble des sujets actifs qui s'opposent au libéralisme des économistes, donc se détachent de l'emprise de l'entourage royal. Il amplifie son importance de manière plus rationnelle par la transformation du langage privé en un langage public, donc d'intérêt général, centré sur la loi à partir des affaires judiciaires. Affaires dont nous savons qu'elles sont prises en main par des médiateurs, en l'occurrence les 
avocats qui s'autorisent à publier des mémoires de facture politique (Maza, 1993) et permettent ainsi d'envisager l'existence d'un corps politique de la nation (Bell, 1994).

Par ailleurs, la résistance parlementaire aux édits de 1776 - édits qui visent explicitement une part importante des privilèges d'Ancien Régime et veut donner l'image d'un roi « législateur absolu » (Turgot), donc indépendant de la médiation des magistrats entre les sujets et la loi - met fin à l'espoir réformateur de constituer un espace civique autour du pouvoir exécutif royal. Certes l'affrontement, au sein des élites, entre réformateurs et conservateurs ne se fait pas sans reformulations et concessions réciproques au sein de stratégies discursives complexes, ainsi que l'ont montré Denise Maldidier et Régine Robin (1974) dans leur étude confrontant les édits de Turgot et les remontrances du Parlement de Paris. Ainsi, autour de la notion de liberté, s'opère un dédoublement très stratégique sur la base de l'affrontement entre une «bonne " et une "mauvaise " liberté : à partir des mêmes syntagmes, voire des mêmes énoncés, les parlementaires exaltent la «bonne» liberté des règlements d'Ancien Régime quand Turgot défend la "bonne" liberté des économistes. Cependant, la crise parlementaire récurrente épuise le fait de la médiation, et renvoie à l'invention, devenue nécessaire en esprit, de la représentation nationale avant même l'affirmation en 1789 d'une immanence et d'une transcendance souveraine de la nation hors de l'emprise royale.

1776, c'est encore l'année où Mably publie De la législation, ou Principes des lois. Dénonçant « la pauvre politique » qui « se fait illusion à elle-même » avec ses remèdes inefficaces, ce penseur majeur franchit un pas de plus. Il ne s'agit plus de faire appel à des juristes pour concrétiser la loi parmi les sujets, mais de trouver une figure représentative de la société elle-même. C'est ainsi que Mably promeut, avec d'autres philosophes, la figure du législateur détenteur de la science politique et de l'art politique. Si « la science politique » tient désormais à des principes fixes issus de la morale, de la loi, et en fin de compte du droit naturel, « la politique moderne », en cherchant à substituer à « la politique de la nature » des projets pernicieux, est condamnée à l'échec (Gauthier, 1995).

A vrai dire, l'affirmation de la nécessaire construction d'un ordre social situé dans la continuité de l'ordre naturel a pour corollaire la méfiance vis-à-vis de toute approche systémique et physicaliste, fortement présente chez les Physiocrates. Il s'agit non pas de déduire les principes des faits, sur un fonds de cartésianisme vulgarisé dans l'idée d'évidence, mais d'observer la nature de l'homme, sa relation aux objets extérieurs et à autrui selon un « ordre local » qui met l'accent sur l'autonomie individuelle au sein même de la réciprocité humaine. S'il est certes nécessaire de «multiplier les faits et les observations de l'expérience" selon Helvétius (De l'Homme, 1773), donc de promouvoir les faits au détriment de l'évidence, c'est au nom des principes d'une morale en acte, donc apte à signifier « comment l'esprit agit ». 
Observer l'homme dans la continuité de l'ordre naturel vers l'ordre social consiste alors en désigner sa finalité, "le bonheur ", et à se donner les moyens d'y parvenir, par la promotion de "l'art social ». Le travail de l'esprit humain s'avère ainsi immense, qui plus est lorsqu'il s'agit de la tâche de son protagoniste principal en matière de politique, le législateur.

1776 c'est enfin l'année de la parution, suivie de sa traduction française, de la Richesse des Nations d'Adam Smith, dix ans après la publication de l'ouvrage majeur d'Adam Ferguson, l'Essai sur l'histoire de la société civile. Avec un tel aboutissement théorique de la lignée des penseurs empiristes anglo-écossais (Gautier, 1993), l'invention de la société civile prend corps de manière particulièrement significative. Elle va en effet de pair avec un individualisme moral qui, tout en affirmant la puissance originelle de l'individu, met, avec la mise en avant du sentiment de sympathie dans la Théorie des sentiments moraux, le « souci de l'autre » au premier plan. A l'interrogation métaphysique sur la nature de l'homme, de facture fortement sensualiste sous l'influence de Condillac, s'ajoute une interrogation anthropologique sur l'homme, être social par nature et la formation de l'ordre social.

C'est alors au cours des années 1780 que se précisent les voies de passage d'une sociabilité civile attestée à l'invention politique. Nous comprenons pourquoi l'expression sieyèsienne de "métaphysique politique " s'avère d'un précieux apport pour situer les fondements de «la nouvelle langue politique». En premier lieu, il s'agit de préciser comment devient possible un discours sur l'utilité de «l'ordre social» qui prend nom, sous la plume de Sieyès, de "sociologie». Sur cette base sociologique se précise alors la capacité des auteurs à penser et à réaliser conjointement de nouveaux objets socio-politiques dont ils reconnaissent l'existence au sein des relations de réciprocité entre les individus singuliers, en les appréhendant à travers des notions compréhensibles et prédictibles telles que la société, l'ordre social, le tout, etc. Par ailleurs, ce discours réflexif, véritable théorie pratique tentée par la désignation de son contenu sous le terme de "socialisme » (Branca-Rosoff, Guilhaumou, 2003), équivalent éphémère d' "art social», systématise, schématise des croyances intentionnelles, au-delà des mœurs usuelles. Il typifie donc des manières nouvelles de penser et d'agir en commun dans le but d'ouvrir la voie à la formation d'un espace public de réciprocité et de dégager le temps à venir de l'agir politique. Un tel espace/temps de l'intersubjectivité et de l'intercommunication des individus libres, en inscrivant des notions-concepts à l'horizon du pensable, ouvre à de nouvelles expérimentations politiques, qui permettront en 1789 de traduire « la métaphysique politique » des objets sociaux dans " un sens commun de la politique " par le fait de l'avènement de " porteurs de vérité », essentiellement les législateurs ${ }^{6}$.

\footnotetext{
${ }^{6}$ Sur l'apport décisif de ce nouveau sens commun dans la réalité d'expérimentations nouvelles inscrites à l'horizon du droit naturel déclaré, voir Gauthier (1992).
} 
Qu'en est-il plus précisément du projet notionnel des penseurs de cette période?

A l'horizon de l'invention de la société (Baker, 1992 ; Kaufmann, Guilhaumou, 2003), il convient d'abord de prendre en compte le point de vue nominaliste d'une nouvelle génération de penseurs qui met l'accent sur l'individu et son jugement, sur le principe d'activité qui le fonde. Déplacé en position seconde par rapport à un principe constituant marqué par la volonté et l'action, le principe de liaison des idées et des signes, si cher à Condillac, permet cependant d'accéder aux vérités utiles à l'horizon d'une métaphysique réduite à la seule requalification de la connaissance par le fait de la référence expérimentale, à l'exemple de la métaphysique de Sieyès (Guilhaumou, 2003). Qui plus est, comme l'a bien précisé Sophia Rosenfeld (2001), il se met alors en place un modèle épistémologique qui donne un statut actif aux signes par le fait d'un langage d'action permettant d'expérimenter le nouvel « ordre social» dans un lien étroit à «l'ordre naturel».

A la différence des Encyclopédistes, ces philosophes pratiques ne croient donc plus dans l'activation d'un principe pensant originaire dans le but de faire usage du raisonnement au sein de l'opinion publique, et donc de réduire les préjugés, en particulier populaires. Ces penseurs et les figures qu'ils proposent - qu'il s'agisse d'Helvétius et de l'observateur-philosophe, de Mably et du législateur, de d'Holbach et de la "science des mœurs ", de Sieyès et de la "métaphysique politique " - s'en tiennent à l'affirmation de nouveaux principes en tant que référents naturels de l'action et de la connaissance, promouvant de fait l'usage de l'artifice pour permettre à la pensée d'accéder à la libre disposition d'objets notionnels inédits sur la base d'un approche non essentialistes des mœurs sociaux. Ils mettent bien au centre de l'activité humaine le travail de l'esprit politique qui permet tout autant d'affirmer qu'il existe « un peuple raisonnable » (Mably) que de façonner les contours de « la classe politique » (Sieyès) apte à constituer un nouvel « ordre social » par la pratique de «l'art social». Ainsi se concrétise une " métaphysique politique » qui trouve son point d'aboutissement dans la radicalité de 1789 grâce à une nouvelle génération de futurs députés, certes moins connus que Sieyès, mais qui opèrent de concert une véritable « révolution de l'esprit » selon l'expression particulièrement juste de Timothy Tackett (1997). Révolution qui s'exprime aussi, à l'encontre de la politique des réformateurs royaux, dans la catégorie d' « antipolitique » (Viola, 1993).

\section{II - De l'avènement de la nation à la proclamation de la République (1789- 1792)}

\section{1- 1789, « L’Année de la Révolution »? 2-}

\footnotetext{
${ }^{7}$ Expression de Sebastien Mercier dans les Annales politiques du 31 décembre 1789.
} 
Du moment 1789, nous retiendrons d'abord, avec l'invention sieyèsienne du mot et de la réalité d' "Assemblée Nationale " à l'horizon de la citoyenneté agissante, et donc du système représentatif, la traduction de la «métaphysique politique », dans des notions telles que « Tiers-Etat », " Assemblée Nationale », " pouvoir constituant», « constitution », etc. sous la forme de vérités pratiques et reconnues. Le terme de "nation», et son pendant nominaliste, la figure de l'individu-nation se trouvent au centre d'un tel dispositif ${ }^{8}$. Ils tiennent leur importance de leur déploiement progressif au $18^{\text {ème }}$ siècle, avec un effet accélérateur à partir du moment où les citoyens peuvent réellement constituer une nation, comme l'a montré David Bell (2001).

Mais c'est la figure du législateur, adepte d'un «art social » basé sur le socle social des «besoins humains » et de leur expérimentation, en référence à une sociologie des « rapports sociaux », qui prend le plus de relief. 1789 est ainsi le point d'aboutissement d'une réflexion pratique sur l'aptitude du législateur empirique à incarner la nouvelle synthèse nationale, en construisant le " tout " de la nation sur une base sociologique, c'est-à-dire à partir des besoins sans cesse renouvelés des individus. Un tel lien constituant entre le politique et le sociologique, spécifique de l'unité sociale en révolution, circonscrit bien le socle de la culture politique française (Rosanvallon, 2004). Mais il n'en prédétermine pas pour autant les potentialités démocratiques ouvertes par les événements révolutionnaires inscrits à l'horizon des droits de l'homme et du citoyen.

En effet 1789 est aussi le point de départ d'un processus révolutionnaire sous la double modalité de la mise en avant de la notion d' "événement ", nouveau champ d'expérience balisé par les «publicistes patriotes» dans le contexte de " la prise de la Bastille », étudiée par Hans-Jürgen Lüsebrink et Rolf Reichardt (1990) et de la notion de « droits déclarés, exposés, reconnus, réalisés », nouvel horizon d'attente de toute action révolutionnaire (Gauthier, 1992). Alors la Révolution française prend, aux yeux de ses acteurs, une tournure soudaine, voire « surnaturelle " ${ }^{9}$, donc de nature fortement pragmatique : elle développe sa qualité expérimentale - de nature foncièrement ontologique nous l'avons vu instaurant ainsi une nouvelle expérience de la temporalité ${ }^{10}$.

La mise en place des antagonismes entre les couples notionnels « lumières/ténèbres » (Reichardt, 2003), "révolution/ancien régime » ${ }^{11}$ s'inscrit dans une même conjoncture. Avec la Déclaration des droits de l'homme, le

\footnotetext{
${ }^{8}$ Voir le chapitre II, la naissance d'une nation, de notre ouvrage L'avènement des porteparole (1998).

9 Pour Grégoire et les «prêtres patriotes » en 1789, La Révolution française réalise les promesses de l'Ecriture. Le moment de l'invention de la nation est aussi « le moment de la régénération ", notion-concept destinée à une brillante carrière révolutionnaire. Voir Hermont-Belot (2000).

${ }^{10}$ Après Reinhart Koselleck (1990), Lynn Hunt (2003) a mis en avant l'importance d'une telle expérience renouvelée de la temporalité.

${ }^{11}$ Voir, dans une perspective comparative franco-allemande, Reichardt (1997).
} 
travail de l'esprit politique n'est plus une simple opération référentielle de production d'objets notionnels nouveaux, il se complexifie par le fait d'un travail déclaratoire de nature foncièrement performative, donc ouvert à de nouvelles expérimentations (Fauré, 1997).

Aux actes de l'esprit politique s'ajoutent, si l'on peut dire, les actes en tant que tels de «la nouvelle langue politique ». Ainsi, au titre de la «souveraineté nationale » et de "la loi au centre », liées dans une perspective pragmatique légitimée par la relation permanente entre le législateur et l'opinion publique, un dispositif argumentatif apparaît autour de l'acte de souveraineté et de l'acte de dire le droit, c'est-à-dire de faire parler la loi détenu par tout citoyen (Guilhaumou, 1998).

Mais, dans l'ensemble des notions mises en place au cours de l'année 1789, qui sont autant de points de passage obligatoire du processus révolutionnaire, des points de repos s'intercalent, laissant ainsi ouvertes de nouvelles potentialités démocratiques. Ainsi en est-il de la notion de " peuple » : garant symbolique du « mouvement politique », elle n'en demeure pas moins largement indéterminée, comme l'a montré Raymonde Monnier (2002) ${ }^{12}$. Quant à la notion de " constitution », Michel Pertué (2003) précise qu'il en est de même, tant dans son rapport à «l'ancien régime » qu'à « la révolution », par le fait de s'énoncer conjointement dans des discours antagonistes, à défaut de pouvoir incarner un norme impérative et intemporelle.

Mises en contexte par leur rôle référentiel, puis mises en acte par leur valeur performative, les notions-concepts de la Révolution peuvent alors réaliser pleinement leurs potentialités démocratiques par leurs mises en argument. Tel est l'enjeu du moment républicain, qui se déploie bien avant la proclamation de la République, en septembre 1792.

\section{3- La République avant la République ${ }^{13}$.}

Retenons, comme significative de la dimension fortement linguistique du moment républicain, l'injonction suivante d'un citoyen anonyme dans le Mercure National du 14 décembre 1790 : «La langue française doit éprouver en même temps que l'empire la révolution qui doit la régénérer"; il ajoute que cette langue dont être enfin "digne d'un peuple-roi ». En ouverture d'un dictionnaire contextuel (Guilhaumou, 2004a), un tel déplacement de la notion de « roi » vers celle de « peuple» contribue à susciter une conscience linguistique de la quête nécessaire d'une " classification juste » des mots. Le " grammairien patriote " Urbain Domergue, créateur de la Société des amateurs de la langue française propose alors, avec l'aide de révolutionnaires aussi prestigieux que

\footnotetext{
${ }^{12}$ Voir aussi le cas de Sieyès présenté par Jacques Guilhaumou et André Tyrsenko dans Desbrousses H., Peloille B., Raulet G. éds (2003).

${ }^{13}$ Le potentiel du républicanisme français dans la perspective d'une reconceptualisation de la théorie politique a été signalé par Philip Pettit (2004).
} 
Brissot, Robespierre, Condorcet et d'autres, la formation d'un « Dictionnaire vraiment philosophique », donnant ainsi à la notion de "régénération » une résonance linguistique. Il participe au mouvement régénérateur qui permet d' " élever notre langue à la hauteur de notre constitution », donc à l'horizon de la pleine et entière réalisation des droits, contribuant ainsi à la fixer comme langue politique ${ }^{14}$.

Au sein de l'espace public de discussion ainsi instauré, et amplifié par le relais des sociétés fraternelles et populaires (Monnier, 1994, 2001, 2003), la mise en argument des notions de " démocratie ", " république ", " liberté ", " égalité », etc. constitue alors une véritable norme rhétorique, amplifiée par des auteurs républicains tels que Lavicomterie, Robert, Bonneville et d'autres. Raymonde Monnier (2002) a également montré que cette rhétorique notionnelle se radicalise, de la fuite du roi au massacre du Champ-de-Mars, dans le va-et-vient constant entre le théoricien publiciste, à l'exemple de Lavicomterie, et le journaliste patriote à l'exemple de Carra. Une telle mise en argument, dans le cadre d'une libre communication des opinions, s'introduit jusqu'au monde de la gaieté : il s'agit de faire rire " dans le sens de la Révolution »- donc de manière anti-aristocratique à l'encontre des publicistes royalistes, qui usent du «bon sens » pour tourner en ridicule les « raisons abstraites » des patriotes - tout en témoignant d'un «état de civilisation » où « la guerre de mots » prend la place de la guerre réelle, comme l'a montré Antoine de Baecque (2000). Cependant, en province, cette mise en argument est surtout amplifiée par la multiplication des porte-parole, à l'exemple des " missionnaires patriotes » marseillais, qui parcourent villes et campagnes au nom de la «Constitution» (Guilhaumou, 1992).

L'été 1792 marque un tournant notionnel dans le moment républicain avec l'apparition de nouvelles potentialités d'usage de la notion de "souveraineté » au-delà du fait attesté, au titre du pouvoir constituant, de «la souveraineté nationale ", expression la plus immédiate de la souveraineté délibérante. L'acte de souveraineté lui-même est d'abord au centre de l'argumentation jacobine autour de «la révolution du 10 août », légitimée par la notion de «mouvement populaire ». Une manière d'être du peuple, sous l'expression « le peuple s'est levé », coïncide désormais avec la réalité plus abstraite de «la langue du peuple" sous l'égide du législateur naturel - un législateur naturellement reconnu par le peuple avant même d'être empiriquement élu - dont Robespierre est le prototype. Désormais, chaque citoyen prend nom de peuple dans tout événement où il participe, autant comme spectateur que comme acteur, et agit au nom du droit naturel déclaré et réalisé (Guilhaumou, 1989a).

${ }^{14}$ Voir le chapitre VIII sur le 'grammairien-patriote' dans notre ouvrage L'avènement des porte-parole (1998). 


\section{III - Apogée et déclin du mouvement jacobin ${ }^{15}$.}

Nous entrons alors de plein pied, avec «l'épiphanie républicaine" propre à l'ouverture de la Convention les 21 et 22 septembre 1792 (Dupuy, 1994), dans le moment jacobin, ne serait-ce que par le fait constamment attesté d'un renouvellement permanent des expressions en usage dans le cours d'un événementiel particulièrement chargé de l'automne 1792 à l'été 1793. Ainsi en est-il, par exemple, du trajet discursif sur la question des « subsistances », qui nous mène du grand débat à la Convention de l'automne 1792, sous couvert d'une "loi générale sur les subsistances », à la mise à l'ordre du jour de la terreur, en septembre 1793, selon l'injonction de Chaumette « Du pain et pour en avoir force à la loi » (Guilhaumou, 2000).

De cet événementiel, nous retiendrons, en matière de mouvement, le temps de l'insurrection des premiers mois de 1793 où se croisent émeutes sur les subsistances, révolte fédéraliste et insurrection proprement dite par un jeu complexe de notions, plutôt que le temps de la mise à l'ordre du jour de la terreur pendant l'été 1793 dont nous avons abondamment rendu compte dans nos travaux autour de la mort de Marat (Guilhaumou, 1989b).

\section{1- Le poids discursif de l'événement : l'insurrection à l'ordre du jour.}

Alors que se multiplient les « désordres populaires " sur les subsistances, avec leur point culminant pendant " la journée du 25 février », les Révolutions de Paris donnent la définition suivante de l'insurrection : " Une insurrection est un combat entre les opprimés et les oppresseurs, mais non entre les riches et les pauvres » au titre de la prééminence de la quête de liberté. Un récit configurant l'événement se met alors en place autour du terme insurrection.

Les Jacobins sont conscients, face au mouvement sectionnaire véhiculant le mot d'ordre, "il faut que le peuple ait du pain », qu'ils ne peuvent empêcher, y compris en leur sein, l'appel à « l'insurrection nationale » alors que « le moment est venu de sauver la patrie » se dit-il à la séance de leur club le 8 mars ( $L a$ Quotidienne). Ainsi, le 10 mars, l'orateur de la députation de la section de la Cité s'adresse à la Commune pour annoncer qu' « elle s'est déclarée en état d'insurrection permanente ». Des membres de la Commune, étonnés d'un tel propos sur l'insurrection, lui demande de préciser : l'orateur ajoute alors qu'il entend par «insurrection armée», "permanence armée », " activité armée » selon le Courrier Français et le Journal de la Révolution de 1792. C'est le début

15 Nous distinguons le mouvement jacobin, en prise directe sur les événements, du jacobinisme : l'impact de ce dernier ne se limite pas à une moment dans la mesure où il correspond à une sensibilité qui traverse l'ensemble de la Révolution française, comme le montre bien Patrice Higonnet (1998). Pour notre part, nous avons proposé une approche du jacobinisme selon notre perspective d'historien marxiste (2002b). 
d'une chaîne d'expressions constitutives du concept de révolution permanente ${ }^{16}$, avec son point culminant au cours des journées révolutionnaires des 31 mai, $1^{\text {er }}$ et 2 juin (Viola, 1989).

La position de la province, et tout particulièrement des Marseillais, renforce tel contexte de radicalité insurrectionnelle (Guilhaumou, 1992). Dans leur adresse à la Convention du 17 mars, accompagnée d'une lettre aux «mandataires infidèles » et d'un appel à " tous les vrais républicains », les jacobins marseillais s'exclament tout d'abord : "Levez-vous : la Patrie est en danger !». Puis ils font appel à « leur droit partiel de souveraineté » pour demander que le peuple " se lève pour la dernière fois" contre les appelants, donc à l'encontre les députés girondins (Guilhaumou, 1992). La réaction de la Convention le 21 mars est franchement hostile: elle improuve une adresse qui vise à "établir le fédéralisme » par le fait de "détruire la représentation nationale, l'unité et l'indivisibilité de la république " (Mercure Universel). Mais la victoire des républicains modérés sur les patriotes radicaux à Marseille tend à effacer provisoirement cette manifestation de fédéralisme jacobin et à focaliser l'attention de la Convention sur «la contre-révolution» issue de «la révolte fédéraliste ». Dans le manifeste, intitulé Marseille aux républicains français, du 12 juin, les fédéralistes sectionnaires veulent se donner les moyens d'action (« C'est le courage des actions qu'il nous faut») en se déclarant " en état légal de résistance à l'oppression » contre «les factieux» de la Convention, en l'occurrence la Montagne ${ }^{17}$. Ils se réclament ainsi, par la voix de la section 24, de «la souveraineté relative, et pour ainsi dire de localité » dans l'exercice de leur « droit de résistance à l'oppression » ${ }^{18}$.

Face à une telle "insurrection partielle » d'une partie de la province (Hanson, 2003), le débat sur la définition et la manière d' " organiser l'insurrection » de manière unitaire domine la scène parisienne. Dès le 18 mars, Chaumette, procureur de la Commune de Paris, définit la « sainte insurrection » comme le " mouvement général du peuple pour assurer la plénitude de ses droits" (Nouvelles politiques). Lui-même et Hébert s'en prennent, tant au club des Cordeliers qu'à la Commune, aux « détracteurs de la sainte insurrection ", en l'occurrence les républicains modérés. Mais ces derniers interviennent sur le terrain de leurs adversaires, l'insurrection elle-même. Par la voie des Annales Patriotes et Littéraires du 30 avril, ils constatent que « Paris est toujours en état d'insurrection » et que les autorités "semblent même approuver le mouvement révolutionnaire par lequel une section du peuple se met au dessus des lois ». Ils argumentent alors que si «l'insurrection est provoquée par les deux partis » qui se disputent la France, elle « aurait pour but d'en écraser un pour faire triompher

\footnotetext{
${ }^{16}$ Faut-il souligner l'importance que ce concept a dans la lecture marxiste de la Révolution française ?

17 Archives départementales des Bouches-du-Rhône, L 2011 bis.

${ }^{18}$ Extrait affiché de la délibération du 7 mai de la section 24, Archives départementales des Bouches-du-Rhône, L 1971.
} 
l'autre, ou elle serait dirigée contre tous les deux », ce qui oblige à se poser la question «Que faut-il donc faire ?». A mi-chemin entre les partis, le Journal de Lyon, dans sa rubrique du 2 avril sur «Un mot à ceux qui veulent une insurrection », propose de s'en tenir à « une insurrection d'opinions ».

Alors que la famille d'insurrection s'augmente ainsi de insurrectionnaire, insurrectionner, insurrectionnel, insurrecteur (Geffroy, 1977), l'insurrection proprement dite commence le 30 mai. L'état d'insurrection de sections parisiennes se répercute immédiatement à la Convention ${ }^{19}$, donc dès l'ouverture de la séance du 31 mai à l'annonce par le président que « de grands mouvements ont lieu dans la ville de Paris " alors qu'un député s'inquiète " qu'une section s'est dite en insurrection ». L'intervention du procureur syndic du département de Paris clarifie la situation : "Le mouvement extraordinaire qui se manifeste dans la ville de Paris doit être considéré comme une insurrection morale ». La démarche légitimée par la Convention des sections en permanence permet alors aux Montagnards d'affirmer que « le peuple fera pour sa liberté un insurrection tout entière » (Danton), au titre de la mise en place d'un processus de révolution permanente. Le lendemain $1^{\mathrm{er}}$ juin, Barère précise alors qu' « il ne faut jamais faire le procès aux révolutions ». Tout en reconnaissant qu' « un grand mouvement s'est fait dans Paris », il martèle le mot de liberté, y compris la liberté des opinions, pour bien marquer qu'il s'agit d'une insurrection en harmonie avec sa concrétisation, la formation d'une nouvelle «Constitution républicaine " dont la Convention conserve l'initiative à l'encontre de toute forme de fédéralisme. Avec la nouvelle déclaration des droits de 1793, Révolution et Constitution participent d'un même mouvement: «Un peuple a toujours le droit de revoir, de réformer, et de changer sa Constitution ». Le droit à l'insurrection s'y inscrit donc naturellement : "Quand le gouvernement viole les droits du peuple, l'insurrection est, pour le peuple et pour chaque portion du peuple, le plus sacré des droits et le plus indispensable des devoirs $»^{20}$.

\section{2- La synthèse nationale sous l'égide du « côté gauche ».}

Une fois le droit à l'insurrection inscrit dans la Constitution, puis la terreur mise à l'ordre du jour à l'initiative du "mouvement révolutionnaire ", la révolution permanente, lexicalement désignée par l'usage répétée de l'expression « en permanence » dans les assemblées délibératives, et plus particulièrement dans les sections, s'inscrit au cœur des événements révolutionnaires. Mais ce n'est vraiment qu'avec la mise en acte de la loi, sous la direction d'un « mouvement national " dirigé par la Convention que se déploie la synthèse nationale aboutissant, en l'an II, à une expérience inédite de construction du «lien social », étendue jusqu'aux institutions les plus proches des citoyens, en

\footnotetext{
${ }^{19}$ Nous suivons la présentation des débats dans les Archives parlementaires, p. 638 et svt., donc sur la base du Moniteur principalement.

${ }^{20}$ Articles XXVIII et XXXIV. Pour comparaison avec 1789, voir Fauré (1992).
} 
particulier les municipalités et les comités de surveillance. Il se met ainsi en place une relation permanente entre le centre et la périphérie, entre les législateurs de la Convention, relayés par les représentants en mission, et les " vrais citoyens» qui gravitent autour des officiers des communes et des surveillants des comités révolutionnaires. Il revient ici à Michel Biard (2002) d'avoir montré comment les "missionnaires de la République» prennent le relais des " missionnaires patriotes », revivifiant ainsi le lien Paris-province, et créant de même un espace notionnel totalisant, occupé ainsi par les termes de " gouvernement », " suspects », " morale », " activité », " mouvement », etc. La dynamique ainsi établi entre le citoyen et le tout social réitère le lien originaire entre le politique et le sociologique, tout en le refondant dans un espace institutionnel où la prise en compte de l'ensemble des "besoins réciproques ", donc y compris au sein de la relation femmes-hommes, rend plus visible la notion d'égalité constitutive de l'ordre social, sans pour autant entamer fondamentalement la domination masculine (Guilhaumou, Lapied, 1997) ${ }^{21}$.

C'est alors du jeu complexe entre les notions de "souveraineté nationale" "souveraineté populaire » et "souveraineté locale ", prises sous le regard de «la centralité législative» que ressort une réalité contradictoire, tout en demeurant républicaine. Une part importante du mouvement républicain a fait l'objet, sous la notion de fédéralisme, d'une condamnation par un centre jacobin. Ainsi, tant à l'encontre du " côté droit ", en référence au fédéralisme modéré du printemps 1793, que vis-à-vis de l'aile radicale du mouvement révolutionnaire, en référence au fédéralisme jacobin de l'automne 1793, les jacobins montagnards se situent au centre de la synthèse nationale, par le fait même de se positionner sur " le côté gauche ». S'appuyant sur l'amplification du rapport de la périphérie au centre dans le cadre de «l'exercice de la souveraineté délibérante ", femmes incluses, sur le terrain du vote pour l'acceptation de l'acte constitutionnel pendant l'été 1793 (Aberdam, 2001), ils peuvent ainsi conserver l'initiative face au mouvement révolutionnaire dirigé par les Cordeliers.

Certes l'opposition entre "côté gauche » et côté droit " remonte aux premiers affrontements au sein de l'Assemblée constituante entre patriotes modérés et patriotes radicaux, et tout particulièrement sur la question coloniale étudiée par Florence Gauthier ${ }^{22}$. Cependant il apparaît nettement en l'an II que cette opposition confère une dimension philosophique en révolution à l'espace des notions-concepts, dans la mesure où elle ne relève pas simplement d'une " identité » des contraires dont il conviendrait de résoudre l'opposition par la

\footnotetext{
${ }^{21}$ Voir également les synthèses plus récents sur Genre et révolution, d'un point de vue analytique et conceptuel, par Martine Lapied et moi-même, que nous avons présentées, soit conjointement, soit de manière séparée, dans de récents colloques, dont ceux de l'Université de Paris I (2004) et de l'association Mnémosyne (2005).

${ }^{22}$ En attendant la publication de son travail sur « l'ouverture du colonialisme esclavagiste et ségrégationniste » au début de la Révolution française, présenté lors de son Habilitation (2003), voir Gauthier F. éd. (2002).
} 
promotion du centre, une fois défait le mouvement jacobin. En fait le «côté gauche » occupe un « extrême », par la reconnaissance de l'universalité du genre humain, qui renvoie le "côté droit» à une autre "extrême", d'essence négatrice, l'inhumanité promue par ceux qui préconisent un langage de classe, non seulement au titre de la « division du travail » mais aussi par le fait, dans le cas des colonies, d'une langue du préjugé de couleur qui refuse aux gens de couleur la dignité d'être humain. L'élimination du " côté droit» par la mise à l'ordre du jour de la terreur s'inscrit bien dans un contexte pensé dans les termes d'un affrontement de classes, dont Grégoire, Robespierre et Marat se sont faits les porte-parole à diverses reprises.

Une telle importance de la problématique notionnelle du "côté gauche », qui outrepasse donc le simple fait de la désignation politique, nous permet de comprendre pourquoi le moment jacobin ne peut se résumer par sa phase la plus active, avec «l'insurrection » et la «terreur » imposées par la dynamique du « mouvement révolutionnaire» du «peuple souverain» de 1792 à 1793. A vrai dire, si l'on veut appréhender la dimension synthétique de ce moment, il nous faut partir ici, comme l'a fait très justement Françoise Brunel $(1992)^{23}$, de la proposition de Billaud-Varenne de « créer un caractère national qui identifie de plus en plus le peuple à son constitution ». Un tel caractère propre de la totalité politique permet d'agir au sein d'un «mouvement national» certes par la traduction permanente de la demande populaire, mais sans pour autant laisser se constituer «un pouvoir exécutif révolutionnaire» autonome, toujours susceptible de mener au fédéralisme, et pire encore de nous faire revenir à la tyrannie. Avec les expressions de "gouvernement révolutionnaire" et d' " institutions civiles » se configure désormais un univers notionnel où la part du projet est fondamental, tout en s'inscrivant dans un espace intersubjectif de sentiments universellement communicables, selon la formulation kantienne.

Le projet national du gouvernement l'an II déploie en effet son activité dans l'établissement d' "institutions civiles ». La première institution est l'instruction nationale. Elle inculque, selon Barère, "l'activité révolutionnaire " en tant que "première qualité civique ${ }^{24}$. La seconde concerne l'établissement d'une " vraie bienfaisance » sur la base la « bienveillance réciproque », avec un accent particulier sur le droit à la subsistance ${ }^{25}$. Il s'agit ici, toujours selon Barère, mais dans son Rapport du 11 mai 1794, de « faire tourner la révolution au profit de ceux qui la soutiennent ». Enfin une troisième institution s'avère tout aussi décisive, l'instauration du français national (Balibar 1985, Branca-Rosoff, 2001), au terme d'une réflexion sur le partage entre la langue française et les

${ }^{23}$ Cette chercheure a également décrit l'univers notionnel de Billaud-Varenne dans son édition crique des Principes régénérateurs du système social, Paris, Publications de la Sorbonne, 1992.

${ }^{24}$ Voir l'extrait de son rapport du $1^{\text {er }}$ juin 1794 reproduit dans Dominique Julia (1981), p. 56.

${ }^{25}$ Duprat (1993), p. 289 et svt. 
idiomes non-français ${ }^{26}$. De Grégoire affirmant que «l'unité de l'idiome est partie intégrante de la révolution ", dans son Rapport au Comité d'instruction publique du 4 juin 1794, à Barère s'exclamant «Révolutionnons donc aussi la langue » dans son Rapport sur les idiomes du 27 janvier 1794, un programme politique d'universalisation et d'uniformisation de la langue française s'impose jusqu'à nos jours (Schlieben-Lange, 1996).

Enfin, d'une institution à l'autre, la notion de « fraternité » joue un rôle essentiel dans la mesure où, sorte de main invisible, elle est le garant de l'harmonie sociale, donc d'un ordre social tendu entre les contraintes de la lutte immédiate et la part nécessaire du projet. C'est aussi le temps, où des notions pratiques, à l'exemple de celle d'hospitalité (Wahnich, 1999), prennent toute leur dimension civique marquant ainsi la concomitance du mouvement des institutions et des principes qui le fonde.

En l'an II, l'activité révolutionnaire touche bien à son terme ultime en légitimant le projet politique basé sur l'idée moderne de nation (Schnapper, 2003). Nulle surprise donc si le transfert notionnel entre législateurs philosophes français et spectateurs philosophes allemands prend alors un essor considérable, à l'exemple des notion de "révolution » et de «nation » (Lüsebrink, Reichardt 1997) dans le cadre plus large des transferts culturels franco-allemands.

\section{4- Le moment thermidorien.}

Qui plus est, le lien paradoxal du centre aux extrêmes permet de penser la continuité du mouvement des notions-concepts du moment conventionnel au moment directorial, au-delà de l'échec du mouvement jacobin avec la chute de Robespierre en Thermidor an II. Ainsi, en proposant la notion historiographique d' " extrême centre » pour qualifier le point aveugle du moment directorial, Pierre Serna $(2003,2005)$ a bien saisi en quoi le mouvement porté par la promotion de la notion de " pouvoir exécutif» depuis le début de la Révolution française élimine progressivement la rhétorique du devoir-être, propre aux penseurs jacobins, au profit d'une maîtrise rhétorique du possible qui détourne des formes notionnelles de la radicalité révolutionnaire (" classe (mitoyenne) », « (parti de la) constitution », " ordre (public) », " tyrannie (du gouvernement) », " anarchie (démagogique) », etc.) pour mieux valoriser un espace modéré. Marc Deleplace (2000) a décrit par ailleurs la matrice lexicale du revers d'un tel " ordre public », sous la forme d'un " désordre " propre à "l'anarchie » et aux " anarchistes », et appréhendé d'abord à travers sa mise en cohérence pendant le moment thermidorien, puis dans sa caractérisation de plus en plus sociale pendant le moment directorial. Quant à Sophie Wahnich (2003), elle a montré en

\footnotetext{
${ }^{26}$ « Dans son projet d' ' uniformiser le territoire' et de rassembler la Nation, la Révolution ne peut manquer de s'interroger sur les différences qui séparent et traversent le pays réel », et tout particulièrement sur le plan linguistiques, soulignent Dominique Julia et Jacques Revel dans leur postface à la réédition de Certeau, Julia, Revel (1975/2002).
} 
quoi la confusion entre les notions d' "ordre social » et d' " ordre public ", pratiquée par les thermidoriens, suscite un refoulement de la demande populaire, et surtout de son régime émotif, hors de l'espace public.

Du moment politique thermidorien, en tant que prélude notionnel au Directoire, retenons aussi l'inversion de «la terreur à l'ordre du jour» en "l'horreur à l'ordre du jour », selon un processus discursif décrit initialement par Bronislaw Baczko (1989), mouvement notionnel qui tend à rompre la dialectique jacobine de la lutte contre les ennemis de la Révolution et de l'installation du gouvernement révolutionnaire, certes à l'avantage d'une radicalité détournée. Une des conséquences de cette rupture est l'utilisation nouvelle en politique du terme de « réaction », tant en Province qu'à Paris (Clay, 1999 ; Monnier, 1999). Mais, plus largement, c'est surtout le constant ajustement notionnel, d'un débat à l'autre au sein de la Convention, qui attire notre attention grâce au minutieux travail de Yannick Bosc (2000). A partir d'un point notionnel fixe exprimé dans les discours de Paine, qui nous renvoie au positionnement des notions de «lien social », d' " exclusion », de " république », d' " égalité », de " liberté », etc. à l'horizon du droit naturel déclaré, l'étude de la mobilité notionnelle au sein de discours antagonistes, en particulier ceux de Boissy d'Anglas, met en évidence une rupture rhétorique par rapport à 1789 dans le fait même de s'en tenir désormais à la notion d' "ordre social», par contrepoids au "désordre » supposé de l'expérience révolutionnaire. La référence au droit naturel perd son aspect normatif, c'est-à-dire sa dimension de « réciprocité » qu'elle avait depuis le début de la Révolution, au plus grand profit de l'affirmation d'une propriété positive de l'individu égoïste.

Tout cela est bien vite dit, ne serait-ce qu'au regard de l'avancée historiographique de nos connaissances historiques sur le Directoire au cours de ces dernières années (Bourdin, Gainot, 1998). Mais, comme le montre Bernard Gainot (2001), dans son étude de la notion de "démocratie représentative » au sein du mouvement néo-jacobin de la période directoriale, les analyses lexicales, y compris les siennes, portent alors plus sur les désignants socio-politiques que sur les notions-concepts.

Concluons donc succinctement sur les temps de l'ouverture tribunicienne et de l'ultime expression libérale du moment napoléonien. Tout commence par l'insistance rhétorique sur l'immortalité de la "grande Nation» en 1797 (Guiomar, 2003) et se termine par l'identification de Napoléon à la nation sous l'expression «Je suis national » telle que nous la trouvons dans le Mémorial de Saint-Hélène, ouvrage à quatre mains récemment étudié, en analyse de discours, par Didier Le Gall (2003). Dans le champ sémantique de six mots-concepts " patrie », " nation », " peuple », « honneur », " égalité » et « liberté », les affirmations napoléoniennes, "je suis la patrie », " je suis national », je suis "l'élu du peuple», veulent constituer une nouvelle communauté d'hommes répondant aux valeurs de l'honneur, et rendent effective l'unification de cette 
communauté par le fait de l'égalité et de la liberté individuelle. Du «sentiment fondamental » (Condillac), déployé dans « le sentiment du moi » (Sieyès) au « sentiment national » (Napoléon), le parcours nominaliste de la "métaphysique politique " s'inverse en privilégiant non plus l'individu particulier, mais sa dimension la plus globalisante, le «tout». La nation-généralité s'impose en fin de compte sur l'individu-citoyen emblématique de la Révolution française

\section{Références bibliographiques}

Aberdam Serge (2001), L'élargissement du vote entre 1792 et 1795 au travers du dénombrement du Comité de division et des votes populaires sur les Constitutions de 1793 et 1795, Thèse de doctorat, Paris I, dir. M. Vovelle, A paraître au CTHS.

Baczko Bronislaw (1989), Comment sortir de la terreur. Thermidor et la Révolution, Paris, Gallimard.

Balibar Renée (1985), L'institution du français. Essai sur le colinguisme des Carolingiens à la République, Paris, PUF.

Baker Keith (1990), Au tribunal de l'opinion. Essai sur l'imaginaire politique au XVIIIème siècle, Paris, Payot.

Baker Keith (1992), «Enligthenment and the institution of society: notes for a conceptual History », in Melching W \& Velema W. (eds), Mains Trends in Cultural History, Amsterdam-Atlanta, Rodopi, p. 95-120.

Bell David (1994), Lawyers and Citizens. The Making of French Political Elite in Old Regim France, Oxford, Oxford University Press, 1994.

Bell David (2001), The Cult of the Nation in France. Inventing Nationalism, 1680-1820, Harvard University Press.

Biard Michel (2002), Missionnaires de la République. Les représentants du peuple en mission (1793-1795), Paris, CTHS.

Bödeker Hans Erich dir. (2002), Begriffsgeschichte, Diskursgeschichte, Metapherngeschichte, Göttingen, Wallstein Verlag, 2002.

Bourdin Philippe, Gainot Bernard, éd. (1998), La République directoriale, 2 v., ParisClermont, Société des études robespierristes et Centre d'histoire.

Bosc Yannick (2000), Le conflit des libertés. Thomas Paine et le débat sur la Déclaration et la Constitution de l'an III, thèse de doctorat, Université de Provence.

Bouthon Cynthia (1993), The Flour War. Gender, Class, and Community in Late Ancien Regim French Society, Ithaca.

Branca-Rosoff Sonia éd. (2001), L'institution des langues. Autour de Renée Balibar, Paris, Editions de la MSH.

Branca-Rosoff Sonia, Guilhaumou Jacques (2003), «De société à socialisme (Sieyès): l'invention néologique et son contexte discursif 》, in Equipe " $18^{\text {ème }}$ et Révolution », Dictionnaire des usages socio-politiques, fascicule 7, p. 143-180.

Brunel Françoise (1992), 1794. Thermidor. La chute de Robespierre, Bruxelles, Complexe

Brunel Françoise, Guilhaumou Jacques (1991), «Les moments de la Révolution française et la synthèse politique », in Recherches sur la Révolution (Vovelle, 1991), p. 45-51.

Brunot Ferdinand (1967), Histoire de la langue française, tome IX, La Révolution et l'Empire, deuxième partie Les événements, les institutions et la langue, Paris, Armand Colin.

Certeau Michel de, Julia Dominique, Revel Jacques (1975/2002), Une politique de la langue, Paris, Gallimard. 
Clay Stephen (1999) «Réaction dans le Midi : le vocable de la vengeance » in Guilhaumou, Monnier (2003), p. 157-186.

Cohen Déborah (2004), Le peuple : de l'Autre au différent. La construction des identités individuelles et collectives des classes populaires (France, XVIIIème siècle), thèse de doctorat EHESS, sous la dir. d'Arlette Farge.

De Baecque (2000), Les éclats du rire. La culture des rieurs au $18^{e}$ siècle, Paris, CalmannLévy.

Deleplace Marc (2000), L'anarchie de Mably à Tournon (1750-1850). Histoire d'une appropriation polémique, Lyon, ENSéditions, 2000.

Desbrousses H., Peloille B., Raulet G. éds (2003), Le peuple. Figures et concepts. Entre identité et souveraineté, , Paris, François-Xavier de Guilbert, 2003.

Dupuy Hélène (1991), " Pour une redécouverte du politique à travers les notions-concepts », in Vovelle (1991).

Dupuy Hélène (1994), «L'épiphanie républicaine dans les actes de la séance inaugurale de la Convention », in Michel Vovelle dir., Révolution et République. L'exception française, Paris, Kimé.

Duprat Catherine (1993), Pour l'amour de l'Humanité. Le temps des Philanthropes, tome 1, Paris, CTHS

Equipe « $18^{\text {ème }}$ et Révolution » dir. (1995), Langages de la Révolution, Paris, Klincksieck.

Equipe « $18^{\text {ème }}$ et Révolution» (Françoise Dougnac, Annie Geffroy, Jacques Guilhaumou, Marie-France Piguet, Raymonde Monnier éds.), Dictionnaire des usages socio-politiques (1770-1815), sept fascicules parus (1985-2003), Paris, Champion, collection « Linguistique française $»$.

Fauré Christine (1992), Les déclarations des droits de 1789, Paris, Payot.

Fauré Christine (1997), Ce que déclarer veut dire : histoires, Paris, PUF.

Formigari Lia (1993), Signs, Science and Politics. Philosophies of language in Europe, 17001830, Amsterdam/Philadelphia, Johns Benjamins.

Gainot Bernard (2001), 1799, un nouveau jacobinisme? La démocratie représentative, une alternative à Brumaire, Paris, CTHS.

Gauthier Florence (1992), Triomphe et mort du droit naturel en Révolution, Paris, PUF.

Gauthier Florence (2002), Périssent les colonies plutôt qu'un principe! Contributions à

l'histoire de l'abolition de l'esclavage, 1789-1804, Paris, Société des études robespierristes.

Gauthier Florence et alii., dir. (1995), La politique comme science morale, Colloque Mably, Bari, Palomar.

Gautier Claude (1993), L'invention de la société civile. Lectures anglo-écossaises (Mandeville, Smith, Ferguson), Paris, PUF.

Geffroy Annie (1977), Matériaux pour l'histoire du vocabulaire français, tome 11, 302 pages, Paris, Klincksieck.

Gordon Daniel (1994), Citizens without Sovereignty. Equality and Sociability in French Thought 1670-1789, Princeton, Princeton University Press.

Guilhaumou Jacques (1989a), La langue politique et la Révolution française, Paris, Meridiens/klincksieck

Guilhaumou Jacques (1989b), 1793. La mort de Marat, Bruxelles, Complexe.

Guilhaumou Jacques (1992), Marseille républicaine (1791-1793), Paris, Presses de Science Po.

Guilhaumou Jacques (1998), L'avènement des porte-parole de la République (1789-1792). Essai de synthèse sur les langages de la Révolution française, Lilles, Presses du Septentrion. Guilhaumou Jacques (2000), "Subsistances (pain, bled(s), grains) 》, Handbuch politischsozialer Grundbegriffe in Frankreich 1680-1820, Heft 19-20, Lüsebrink Hans et Reichardt Rolf éds, Munich, Oldenbourg, p.141-201. 
Guilhaumou Jacques (2002a), Sieyès et l'ordre de la langue. L'invention de la politique moderne,Paris, Kimé.

Guilhaumou Jacques (2002b), « Jacobinisme et marxisme : le libéralisme politique en débat », Actuel Marx, $\mathrm{N}^{\circ} 32$, p. 109-124.

Guilhaumou Jacques (2003), «L'avènement de la 'métaphysique politique'. Sieyès et le nominalisme politique », in Kaufmann, Guilhaumou (2003), p. 201-226.

Guilhaumou Jacques (2004a), «Fragments d'un dictionnaire contextuel des mots de la Révolution française », Cahiers de lexicologie, 84, 2004-1, p. 1-16.

Guilhaumou Jacques (2004b), «Agir en révolution: action, émeute, révolte, révolution, mouvement insurrection », in Monnier (2004).

Guilhaumou Jacques (2006 a), «Sieyès et l'invention du terme de sociologie », à paraître.

Guilhaumou Jacques (2006b), Discours et événement. L'histoire langagière des concepts, Paris, à paraître.

Guilhaumou Jacques et Monnier Raymonde dir. (2003), Des notions-concepts en révolution. Autour de la liberté politique à la fin du $18^{\text {ème }}$ siècle, Paris, Société des études robespierristes.

Guilhaumou Jacques, Lapied Martine (1997), «L'action politique des femmes pendant la Révolution française », in C. Fauré éd., Encyclopédie historique et politique des femmes, Paris, PUF.

Guiomar Jean-Yves (2003), «Histoire et signification d'une expression célèbre : la grande nation (août 1797-automne 1799) », in Equipe «18 ${ }^{\text {ème }}$-Révolution », Dictionnaire des usages socio-politiques, fascicule 7, p. 67-82

Hanson Paul (2003), The Jacobin Republic under Fire. The Federalisre Revolt in the French Revolution, Pennsylvania State University Press.

Hermont-Belot Rita (2000), L'abbé Grégoire. La politique et la vérité, Paris, Seuil.

Higonnet Paul (1998), Goodness beyond Virtue. Jacobins during the French Revolution, Cambridge, Harvard University Press.

Hunt Lynn (1992), The Family Romance of the French Revolution, Berkeley, University of California Press.

Hunt Lynn (2003), « The World We Have Gained : the Future of the French Revolution », American Historical Review, February, p. 1-19

Julia Dominique (1981), Les trois couleurs du tableau noir. La Révolution, Paris, Belin.

Kaufmann Laurence (2000), A la croisée des esprits. Esquisse d'une ontologie du fait social : l'opinion publique, Thèse de doctorat, dir. P. Béaud et L. Quéré, Paris EHESS/Lausanne.

Kaufmann Laurence (2004), «Entre fiction et réalité. L'opinion publique dans la France du XVIIIème siècle ", in L'avènement de l'opinion publique. Europe et Amérique XVIII-XIXème siècles, sous la dir. de Javier Fernandez Sebastian et Joëlle Chassin, Paris, L'Harmattan.

Kaufmann Laurence, Guilhaumou Jacques dir. (2003), L'invention de la société. Nominalisme politique et science sociale au XVIIIème siècle, Paris, Editions de l'EHESS.

Koselleck Reinhart (1990), Le futur passé. Contribution à la sémantique des temps historiques, Paris, Editions de l'EHESS.

Lapied Martine, Peyrard Christine dir. (2003), La Révolution française au carrefour des recherches, Aix-en-Provence, Publications de l'Université de Provence.

Le Gall Didier (2003), Napoléon et le Mémorial de Sainte-Hélène. Analyse d'un discours, Paris, Kimé.

Lüsebrink Hans, Reichardt Rolf (1990), Die «Bastille». Zur Symbolgeschichte von Herrschaft und Freiheit, Frankfurt-am-main, Fischer.

Lüsebrink Hans, Reichardt Rolf dir. (1997), Kulturtransfer im Epochenumbruch Frankreich Deutschland 1770 bis 1815, deux volumes, Leipzig Universitätverlag.

Lüsebrink Hans, Reichardt Rolf, Eberhard Schmitt dir. (1985-2000), Handbuch politischsozialer Grundbegriffe in Frankreich (1680-1820), Heft 1-20, Munich, Oldenbourg. 
Maldidier Denise, Robin Régine (1974), «Polémique idéologique et affrontement discursif en 1776: les grand édits de Turgot et les remontrances du Parlement de Paris », in J. Guilhaumou, R. Robin et alii. (1974), Le discours comme objet de l'histoire, Paris, Les Editions ouvrières, p. 13-81.

Martin Jean-Clément (1998), Contre-révolution, Révolution et Nation en France (1789-1799), Paris, Seuil.

Mathiez Albert (1973), La vie chère et le mouvement social sous la terreur, Paris, Payot, deux volumes.

Maza Sara (1993), Vies privées, affaires publiques: les causes célèbres de la France prérévolutionnaire, Paris, Fayard.

Monnier Raymonde (1994), L'espace public démocratique. Essai sur l'opinion à Paris de la Révolution au Directoire, Paris, Kimé.

Monnier Raymonde (1999), «Un mot nouveau en politique : réaction sous Thermidor », in Guilhaumou, Monnier (1999), p. 127-156.

Monnier Raymonde (2001), "Démocratie représentative et république démocratique », Annales Historiques de la Révolution française, 3, p. 1-21.

Monnier Raymonde (2002a), «Républicanisme, libéralisme et Révolution française », in Les libéralismes au regard de l'histoire, Florence Gauthier et Jacques Guilhaumou éds., Actuel Marx $\mathrm{N}^{\circ} 32$, p. 83-108

Monnier Raymonde (2002b), «Autour des usages d'un nom indistinct : peuple sous la Révolution française », Dix-huitième siècle, N³4, p. 389-418.

Monnier Raymonde (2003), "Républicanisme et Révolution française », French Historical Studies, v. $26-1$, p. 87-118.

Monnier Raymonde éd. (2004), Révoltes et révolutions en Europe (Russie comprise) et aux Amériques, Paris, Ellipses.

Pertué Michel (2003), «La notion de constitution à la fin du $18^{\mathrm{e}}$ siècle », in Guilhaumou, Monnier (2003), p.39-54.

Pettit Philip (2004), Républicanisme. Une théorie de la liberté et du gouvernement, Paris, Gallimard.

Reichardt Rolf (1997), «Die Revolution- ein magischer Spiegel' », in Lüsebrink H.-J. et Reichardt R. éd., Kulturtransfer im Epochenumbruch Frankreich -Deutschland 1770 bis 1815, Leipzig Universität Verlag, V. 2, s.883-999.

Reichardt Rolf (2003), «Visualiser la logomachie entre Lumières et Ténèbres ou les étranges métamorphoses de l'éteignoir dans les estampes (1789-1830)», in Guilhaumou, Monnier (2003), p15-38.

Rosanvallon Pierre (2004), Le modèle politique français. La société civile contre le jacobinisme de 1789 à nos jours, Paris, Seuil.

Rosenfeld Sophia (2001), A Revolution in Language. The Problem of Signs in Late Eighteenth-Century France, Stanford, Stanford University Press.

Serna Pierre (2003), " Existe-t-il un 'extrême centre' ? ou le point aveugle de la république directoriale. L'exemple de La Décade », in Guilhaumou, Monnier (2003), p. 149-168.

Serna Pierre (2005), La République des Girouettes. 1789-1815, et au-delà une anomalie politique : la France de l'extrême-centre, Paris, Champvallon.

Schlieben-Langen Brigitte (1996), Idéologie, révolution et uniformité de la langue, Liège, Mardaga.

Schnapper Dominique (2003), La communauté des citoyens. Sur l'idée moderne de nation, Folio/Essais, Paris, Seuil.

Tackett Timothy (1997), Par la volonté du peuple. Comment les députés de 1789 sont devenus révolutionnaires, Paris, Albin Michel. 
Van Kley Dale (1994), The French Idea of Freedom. The Old Regim and the Declaration of Rights of 1789, Stanford, Stanford University Press.

Viguier Anne, "Individu (1770-1830): un processus de politisation du vocabulaire », in Dictionnaire des usages socio-politiques (1770-1815), fascicule 4, sous la dir. de l'équipe «18 ${ }^{\text {ème }}$ et Révolution », Paris, Champion, p. 111-144.

Viola Paolo (1989), Il trono vuoto. La transition delle sovranita nella rivoluzione francese, Turin, Einaudi.

Viola Paolo (1993), Il crollo dell'antico regime. Politica e antipolitica nella Francia della Rivoluzione, Rome, Donzelli.

Vovelle Michel (1993), La découverte de la politique. Géopolitique de la Révolution française, Paris, La Découverte.

Vovelle Michel, dir. (1991), Recherches sur la Révolution, Paris, Société des études robespierristes.

Wahnich Sophie (1999), «Les révoltes de l'hospitalité. Analyse sémantique d'une notion pratique ", Dictionnaire des usages socio-politiques (1770-1815),, sous la dir. de l'équipe « $18^{\mathrm{e} m e}$ et Révolution », fascicule 6, Paris, Champion, p. 31-51.

Wahnich Sophie (2003), «Désordre social et émotions publiques pendant la Révolution française », in Kaufmann, Guilhaumou (2003), p. 227-260. 